\title{
Hoher Nutzen der Thrombektomie
}

Fragestellung: Wie ist der Nutzen der Thrombektomie bei Patienten mit distalen Verschlüssen der A. carotis interna und proximalen Verschlüssen der A. cerebri media?

Hintergrund: Die Standardtherapie ischämischer Insulte war bisher die systemische intravenöse Thrombolyse mit rt-PA. Bei großen Gefäßverschlüssen liegt dabei allerdings die Rekanalisierungsquote unter $50 \%$. Deshalb wurde vor einigen Jahren die Thrombektomie in die Therapie des akuten Schlaganfalls eingeführt, wobei die ersten drei Studien bei Kathetern mit unzureichenden Rekanalisationsraten und schlechtem Studiendesign mit nicht optimaler Patientenauswahl negativ ausgingen. In der Folgezeit wurden dann fast zeitgleich fünf randomisierte Studien durchgeführt, wobei vier dieser Studien nach den Ergebnissen der MR CLEAN-Studie vorzeitig abgebrochen wurden. Das führte dazu, dass für die vier zwar positiven Studien die Fallzahlen relativ gering waren. Daher war es notwendig zur Absetzung des Therapieeffektes eine Metaanalyse der fünf vorliegenden randomisierten Studien durchzuführen.

Patienten und Methodik: Die HERMES-Arbeitsgruppe hatte bereits zu einem Zeitpunkt als die Studien noch liefen, vereinbart, nach Abschluss der Studien eine prospektive Metaanalyse durchzuführen. Die hier publizierte Metaanalyse bezieht sich auf Daten der Studien MR CLEAN, ESCAPE, REVASCAT, SWIFT PRIME und EXTEND IA, die alle zwischen Dezember 2010 und Dezember 2014 durchgeführt wurden. Eingeschlossen wurden in diese Studien Patienten mit Verschlüssen der distalen A. carotis interna und der proximalen A. cerebri media in einem 12-Stundenfenster, wobei in fast allen Studien ein Stent-

Retriever für die Thrombek-

Goyal M, Menon BK, van Zwam WH et al; HERMES collaborators. Endovascular thrombectomy after large-vessel ischaemic stroke: a meta-analysis of individual patient data from five randomised trials. Lancet 2016; 387: 1723 - 31 tomie verwendet wurde. Der primäre Endpunkt war der Wert auf der modifizierten Rankin Skala nach 90 Tagen. Methodisch wurde eine ordinale logistische Regressionsanalyse durchgeführt und es wurde eine Shift-Analyse der modifizierten Rankin Skala berechnet, wobei für die Adjustierung Alter, Geschlecht, Schwere des Schlaganfalls, Lokalisation des Gefäßverschlusses, Gabe von i.v. rt-PA, der ASPECT Score im CT und die Zeit von Beginn des Schlaganfalls bis zur Randomisierung eingingen.

Ergebnisse: In die Analyse gingen die Ergebnisse von 1.287 Patienten ein, wobei 634 thrombektomiert wurden und 653 die Kontrollpopulation darstellten. Das mittlere Alter betrug 68 Jahre, $53 \%$ waren Männer. Die mittlere Schwere des Schlaganfalls bei Behandlungsbeginn lag auf der NIHSS bei 17, $21 \%$ hatten distale Verschlüsse der A. carotis interna, $69 \%$ proximale Verschlüsse der A. cerebri media und $8 \%$ Verschlüsse des M2Segments. Bei $85 \%$ der Patienten erfolgte eine intravenöse Thrombolyse. In der Thrombektomiegruppe betrug die Zeit vom Beginn der Symptomatik bis zur Reperfusion im Schnitt 285 Minuten. Die Odds Ratio für einen guten Outcome betrug 2,26. Einen modifizierten Rankin Score von 0 bis 2 nach 90 Tagen erreichten $56 \%$ in der Thrombektomie- und $26,5 \%$ in der konservativen Behandlungsgruppe. Das entspricht einer absoluten Differenz von $19,5 \%$ und einer adjustierten Odds Ratio von 2,71. Einen Wert auf der NIHSS von 0 bis 2 nach 24 Stunden erreichten $21 \%$ in der Interventions- und $8 \%$ in der Kontrollgruppe. Auch dieser Unterschied war hoch signifikant.

Die number needed to treat für die endovaskuläre Therapie, um die Behinderung auf der modifizierten Rankin Skala um mindestens einen Wert zu verbessern, betrug 2,6. Die Ergebnisse waren konsistent und unabhängig von Alter, ASPECT-Score, rt-PA-Gabe, Lokalisation des Gefäßverschlusses, Schlaganfallschwere, Zeit bis zur Randomisierung, Geschlecht und der Frage, ob eine Tandemläsion (Verschluss der A. carotis interna plus Verschluss der A. cerebri media) vorlag. Die Sterblichkeit nach 90 Tagen und das Risiko parenchymaler Hirnblutungen oder symptomatischer intrakranieller Blutungen unterschied sich nicht zwischen den Therapiegruppen.

Schlussfolgerungen: Bei Patienten mit Verschlüssen der proximalen Arterien in der vorderen Zirkulation hat die endovaskuläre Thrombektomie einen hohen therapeutischen Nutzen. Die Ergebnisse haben klare Implikationen für die Versorgung von Schlaganfallpatienten.

\section{Geeignete Patienten schnellstmöglich thrombektomieren}

Diese Metaanalyse unterstreicht, was die Ergebnisse der fünf Einzelstudien bereits nahegelegt hatten, nämlich einen hochsignifikanten Effekt der Thrombektomie bei Verschlüssen in der vorderen Zirkulation und bei schweren Schlaganfällen. Eine number needed to treat von 2,6 ist hochrelevant. Wichtig ist, dass diese Ergebnisse für praktisch alle Untergruppen von Patienten gelten, wobei ein kritischer Parameter natürlich das Zeitintervall zwischen Beginn der Symptomatik und Zeit- punkt der Rekanalisation ist. Je früher die Rekanalisation erfolgt, umso besser sind die Ergebnisse. Auf Deutschland bezogen heißt dies, dass alle Schlaganfallstationen, die selbst nicht im eigenen Haus thrombektomieren können, eine Partnerschaft mit einer überregionalen Stroke Unit in der Umgebung organisieren müssen, damit Patienten, die für diese Therapie infrage kommen, mit minimalster Verzögerung einem solchen Zentrum zugeführt werden. 